\title{
YOLO-DoA: A lightweight, real-time, and effective implementation for DoA estimation
}

This paper was downloaded from TechRxiv (https://www.techrxiv.org).

\section{LICENSE}

CC BY 4.0

SUBMISSION DATE / POSTED DATE

20-01-2022 / 25-01-2022

CITATION

Si, Chengke; Fan, Rong (2022): YOLO-DoA: A lightweight, real-time, and effective implementation for DoA estimation. TechRxiv. Preprint. https://doi.org/10.36227/techrxiv.18737684.v1

DOI

10.36227/techrxiv.18737684.v1 


\title{
YOLO-DoA: A lightweight, real-time, and effective implementation for DoA estimation
}

\author{
Chengke Si, Rong Fan, Xinyu Zhu and Yihe Wan
}

\begin{abstract}
Existing data-driven methods for direction-ofarrival (DoA) estimation are usually implemented by classifiers with binary vector labels, which suffer from insufficient utilization about features of sources. In this correspondence, an efficient YOLOv3-based approach for DoA estimation named YOLO-DoA is proposed, which is implemented as a detection task to spatially separated angular boxes. DoAs of sources with confidence scores are directly predicted from the spectrum proxy with YOLO-DoA and an end-to-end estimation is realized. By combining squeezeand-excitation operation, cross stage partial connections, and an improved loss for angular box regression, the performance of YOLO-DoA is significantly enhanced. Simulation results demonstrate that the proposed approach outperforms several state-ofthe-art methods in terms of network size, computational cost, prediction time and accuracy of DoA estimation.
\end{abstract}

Index Terms-Direction-of-arrival (DoA) estimation, array signal processing, sparse signal representation (SSR), convolutional neural network (CNN), YOLOv3.

\section{INTRODUCTION}

D IRECTION-OF-ARRIVAL (DoA) estimation is a critical technique in the fields of $5 \mathrm{G}$ wireless communications [1], indoor localization [2], prediction of multi-user multipleinput multiple-output (MIMO) channels [3], etc.

For a long time, DoA estimation is achieved by modeldriven approaches such as multiple signal classifications (MUSIC) [4], beamforming [5] and the estimation of signal parameters via rotational invariance techniques (ESPRIT) [6]. In these methods, the array manifolds are generally approximately built from mathematical perspectives, so their performances are limited by the mismatch of system models. Moreover, the array manifolds established in advance are static, which cannot adapt to the changes of environment.

According to the well-known universal approximation theorem, any continuous function on a compact subset of $\mathbb{R}^{n}$ can be approximated by a deep neural network [7]. Recently, several data-driven methods repurpose classification neural networks to estimate the DoAs of sources, which overcome the drawbacks of model-driven methods. In particular, a hybrid neural network for DoA estimation (i.e., ResNet-DoA) is

Manuscript received January 19, 2022

The work is supported in part by Sichuan Science and Technology Program under Grant 2021 YFG0192 and the National Natural Science Foundation of China under Grant 62061003. (Corresponding author: Rong Fan.)

Chengke Si, Rong Fan and Xinyu Zhu are with the Institute of Electronic and Electrical Engineering, Civil Aviation Flight University of China, Guanghan, 618307 China. (e-mail: sichengke@ cafuc.edu.cn; fanrong@ cafuc.edu.cn; xyzhu@cafuc.edu.cn)

Yihe Wan is with the Jiangxi Province Engineering Research Center of Special Wireless Communications, Jiujiang, 332000 China. (e-mail: wanyihe2007@sina.com). proposed in [8]. Although high accuracy of DoA estimation is obtained by ResNet-DoA, the procedure is complex because it requires independent training of two sub-networks. In [9], a convolutional neural network (CNN) for DoA estimation (i.e, CNN-DoA) is proposed in low signal-to-noise ratio (SNR) case. But it has a large number of network parameters because of several full connected layers utilized. Besides, another CNN-based approach named RF-DoA is proposed in [10], which overcomes the challenge of network size. However, many grouped conventional layers are used in RF-DoA, which reduce the speed of training and prediction. As a matter of fact, existing data-driven methods for DoA estimation are implemented by classifiers with binary vector labels, which cannot exploit the angular features sufficiently. Moreover, the peak-search is required in these methods, which increases the computational overhead and introduces fit-error.

In fact, DoA estimation can be regarded as recovering support of sources from angular features, hence it can also be reframed as an support detection task [11]. Meanwhile, YOLOv3 has been widely used in real-time object detection, which is implemented as a detection task based on bounding boxes [12]. Inspired by above insights, an efficient detection neural network for DoA estimation based on angular box regression is proposed, viz., YOLO-DoA. The backbone of YOLO-DoA is established by imposing cross stage partial (CSP) [13] connections into a pruned ResNet18 [14], which realizes the lightweight and real-time performance. Meanwhile, the generalized intersection over union (GIoU) loss [15] and weighted cross-entropy are both used, which improve the feature extraction ability of network. Besides, the network stability is enhanced by imposing squeeze-and-excitation (SE) operation [16]. Finally, DoAs of sources with confidence scores are derived directly from the outputs of array.

The main contributions of the paper include three aspects. Firstly, as far as we know, it is the first work to estimate DoAs of sources with deep object detection network instead of classification network, which achieves an end-to-end prediction without peak-search. Secondly, an angular box based sample labeling strategy for directions of sources is proposed, which improves the information utilization of sources because areatype rather than line-type labels are exploited. Thirdly, compared to the state-of-the-art data-driven methods, YOLO-DoA has merits of lightweight network size, low computational cost, and high accuracy of DoA estimation.

Notation: The scalar, vector and matrix are denoted by lower case letters, bold lower case letters and upper bold case letters, respectively. $\boldsymbol{\chi}_{(d)}$ denotes a tensor and $(\cdot)^{\top}$ is the transpose operation. Symbols $\mathbb{E}\{\cdot\}$ and $\operatorname{Re}\{\cdot\}$ denote the expectation 
operator and the real part of a complex vector, respectively. Symbol $J$ equals $\sqrt{-1}$. $\mathbf{I}_{M}$ is the $M \times M$ identity matrix. $\lceil\cdot\rceil$ is the rounding up operation.

\section{PROBlem Formulation}

Assuming that $K$ far-field narrow-band sources impinge on a $M$-element uniform linear array (ULA- $M$ ) from distinct directions. The unknown DoAs of sources and the potential space are denoted by $\boldsymbol{\theta}=\left[\theta_{1}, \theta_{2}, \cdots, \theta_{K}\right]^{\top} \in \mathbb{R}^{K}$ and $\theta$, respectively. The $\theta$ is further divided into a discrete direction set $\boldsymbol{\Psi}=\left[\varphi_{1}, \varphi_{2}, \cdots, \varphi_{L}\right]^{\top} \in \mathbb{R}^{L}$, where $L$ is the number of discrete grids about the potential space. Meanwhile, the sparse signal vector is defined as $\tilde{\mathbf{s}}(t)=\left[\tilde{s}_{1}(t), \tilde{s}_{2}(t), \cdots, \tilde{s}_{L}(t)\right]^{\top} \in \mathbb{C}^{L}$. The $l$-th entry $\tilde{s}_{l}(t)$ only has non-zero value when $\varphi_{l} \in \boldsymbol{\theta}$. Then the overcomplete sparse model for DoA estimation can be formulated as [17]

$$
\mathbf{y}(t)=\sum_{l=1}^{L} \mathbf{a}\left(\varphi_{l}\right) \tilde{s}_{l}(t)+\mathbf{v}(t), \quad n=1,2, \cdots, T
$$

where $\mathbf{y}(t) \in \mathbb{C}^{M}$ is the array observation data in the $t$ th snapshot. $T$ is the total number of collected snapshots. $\mathbf{a}\left(\varphi_{l}\right)$ denotes the steering vector corresponding to $\varphi_{l}$ and $\mathbf{a}\left(\varphi_{l}\right)=\left[1, e^{j 2 \pi d \sin \left(\varphi_{l}\right) / \lambda}, \cdots, e^{j 2 \pi(M-1) d \sin \left(\varphi_{l}\right) / \lambda}\right]^{\top}$, where $d$ is the array interelement distance and $\lambda$ is the wavelength of sources. $\mathbf{v}(t)$ is the complex Gaussian white noise with covariance matrix $\mathbb{E}\left\{\mathbf{v}(t) \mathbf{v}(t)^{H}\right\}=\sigma_{v}^{2} \mathbf{I}_{M}$, where $\sigma_{v}^{2}$ is the power of noise. In practice, assuming that all sources are independent and mutually uncorrelated. And thus, the data covariance matrix $\mathbf{R}_{\mathbf{y}}$ can be formulated as

$$
\mathbf{R}_{\mathbf{y}}=\sum_{l=1}^{L} \bar{\sigma}_{l}^{2} \mathbf{a}\left(\varphi_{l}\right) \mathbf{a}^{H}\left(\varphi_{l}\right)+\bar{\sigma}_{v}^{2} \mathbf{I}_{M}
$$

where $\bar{\sigma}_{l}^{2}=\sigma_{k}^{2}$ when $\varphi_{l}=\theta_{k}(k=1,2, \cdots, K)$, otherwise $\bar{\sigma}_{l}^{2}=0$. Noted that the $m$-th column of $\mathbf{R}_{\mathbf{y}}$ can be written as $\mathbf{r}_{m}=\mathbf{R}_{\mathbf{y}} \mathbf{e}_{m}$ from (2), where $\mathbf{e}_{m}$ is an $M \times 1$ vector with the $m$-th element being 1 and others being 0 . Let vector $\mathbf{r}$ denote the vectorized form of $\mathbf{R}_{\mathbf{y}}$, which is

$$
\mathbf{r}=\left[\mathbf{r}_{1} ; \mathbf{r}_{2} ; \cdots ; \mathbf{r}_{M}\right] \in \mathbb{C}^{M^{2}}
$$

Further, we define matrix $\tilde{\mathbf{A}}=\left[\mathbf{A}_{1} ; \mathbf{A}_{2} ; \cdots ; \mathbf{A}_{M}\right] \in \mathbb{C}^{M^{2} \times L}$, where $\mathbf{A}_{m} \triangleq\left[\mathbf{a}\left(\varphi_{1}\right) \mathbf{a}^{H}\left(\varphi_{1}\right) \mathbf{e}_{1}, \cdots, \mathbf{a}\left(\varphi_{L}\right) \mathbf{a}^{H}\left(\varphi_{L}\right) \mathbf{e}_{m}\right] \in \mathbb{C}^{M \times L}$ and $m=1,2, \cdots, M$. Next, we take $\mathbf{x} \triangleq \operatorname{Re}\left\{\tilde{\mathbf{A}}^{H} \mathbf{r}\right\} \in \mathbb{R}^{L \times 1}$ as the spectrum proxy. Thus, a collection of $D$ examples is established. The $d$-th example $\mathbf{x}_{(d)}$ and its corresponding direction $\boldsymbol{\theta}_{(d)}$ are combined in pairs $\left(\mathbf{x}_{(d)}, \boldsymbol{\theta}_{(d)}\right)$ to form the dataset $\mathbb{D}_{\text {data }}=\left\{\left(\mathbf{x}_{(I)}, \boldsymbol{\theta}_{(I)}\right),\left(\mathbf{x}_{(2)}, \boldsymbol{\theta}_{(2)}\right), \cdots,\left(\mathbf{x}_{(D)}, \boldsymbol{\theta}_{(D)}\right)\right\}$. Finally, the DoA estimation is performed from $\mathbb{D}_{\text {data }}$ with different data-driven methods [8]-[10].

\section{YOLO-DOA FOR DOA ESTIMATION}

\section{A. Data Preprocess}

In order to perform the DoA estimation in regression way, each sample in dataset $\mathbb{D}_{\text {data }}$ must be transformed in the form at first. Without loss of generality, assuming that the potential space $\theta$ is in the range $\left[-\theta_{\max }, \theta_{\max }\right)$ and the angle resolution is $\delta$, which makes $L=\left\lceil\left(2 \times \theta_{\max }\right) / \delta\right\rceil$. Further, we equally divide $\theta$ into $S$ spatial subregions (SubRegs) and each SubReg contains $P$ micro regions (MicroRegs). For a specific direction $\theta_{k}$, one angular box is generated, which is defined as $\operatorname{box}_{\theta_{k}} \triangleq\left\{x_{\theta_{k}}, y_{\theta_{k}}, W, H, \kappa\right\}$, where $x_{\theta_{k}}$ and $y_{\theta_{k}}$ are the center coordinates. $W, H$ and $\kappa$ denote the width, height, and confidence score, respectively. And thus, the data label can be formulated as a tensor $\chi \in \mathbb{R}^{S \times P \times 5}$.

Specifically, let $S=24, \theta_{\max }=90^{\circ}$, and $\delta=1^{\circ}$, which makes $L=180$. Hence, we expand example $\mathbf{x}_{(d)} \in \mathbb{R}^{180 \times 1}$ into $\overline{\mathbf{x}}_{(d)} \in \mathbb{R}^{192 \times 1}$ by zero padding for the need of downsampling with a factor of 32. Besides, if direction $\theta_{k}$ falls into a SubReg, that SubReg is responsible for $b^{\circ} x_{\theta_{k}}$. And the index of responsible SubReg is $i=\left\lceil\theta_{k} / 8^{\circ}\right\rceil$ because each SubReg covers a range of $8^{\circ}$. The index of responsible MicroReg is $j=\left\lceil\bmod \left(\theta_{k}, 8^{\circ}\right) / P\right\rceil$. For any box $_{\theta_{k}}$, we assign $x_{\theta_{k}}=\theta_{k}$, $y_{\theta_{k}}=0.5, H=1$ and $\kappa=1$. Constants $W$ and $P$ are determined by experimentally. Meanwhile, the slice $\chi^{i, j,:}$ of label $\mathcal{X}$ is assigned with $b x_{\theta_{k}}$, which is defined as $b o x_{i, j}$. And thus, each $\boldsymbol{\theta}_{(d)}$ in $\mathbb{D}_{\text {data }}$ is transformed into tensor label $\chi_{(d)}$. Then $\overline{\mathbf{x}}_{(d)}$ and $\chi_{(d)}$ are combined in pairs $\left(\overline{\mathbf{x}}_{(d)}, \chi_{(d)}\right)$ to form dataset $\left\{\left(\overline{\mathbf{x}}_{(I)}, \chi_{(1)}\right),\left(\overline{\mathbf{x}}_{(2)}, \chi_{(2)}\right), \cdots,\left(\overline{\mathbf{x}}_{(D)}, \chi_{(D)}\right)\right\}$. Both training dataset $\mathbb{D}_{\text {train }}$ and test dataset $\mathbb{D}_{\text {test }}$ are labeled in this way. The diagrammatic sketch is shown in Fig. 1

It must be pointed out that existing labeling strategy with binary vector only exploits line-type local features to characterize the DoAs of sources. Instead, our labeling strategy with angular box can make full use of area-type features of sources, which improves the utilization of features. Then, the predicted box is generated from the area-type predicted features of sources by YOLO-DoA.

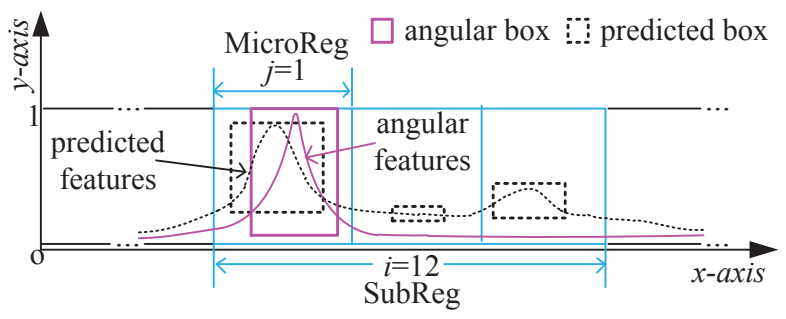

Fig. 1: The diagrammatic sketch about angular box, predicted box, the responsible SubReg and MicroReg for $\theta_{k}=98^{\circ}$.

\section{B. YOLO-DoA Design}

As shown in Fig. 2, a lightweight, real-time, and effective network is proposed in this subsection, which consists of Backbone, Neck, and Head. The convolutional layer (Conv), full connected layer (FC), batch normalization (BN), Sigmoid, the rectified linear unit (ReLU), LeakyReLU, upsampling (Ups), global pooling, and max pooling (Pool) are referred in [18]. SR denotes the residual module with the SE operation [16]. CSR is constructed by two SR modules with CSP connections [13]. Symbol ' $*$ ' is downsampling with stride of $2 \times 1$, otherwise the stride is $1 \times 1$. Ups is upsampling with stride of $2 \times 1$. Notation 'module, $\mathrm{w} \times \mathrm{h}, \mathrm{c}$ ' is the module with kernel size of $\mathrm{w} \times \mathrm{h}$ and channel number of c. ' $\oplus$ ', ' $\otimes$ ' and ' $C$ ' ' are addition, 
Hadamard product and concatenation operation, respectively. The workflow of YOLO-DoA is as follows.

In the Backbone, the multi-stage features of sources are extracted from shallow to deep. The channel number of $\overline{\mathbf{x}}$ is firstly extended by the CBR. And then, downsampling is executed by max pooling. In the subsequent, features of sources are extracted through four cascaded CSRs. In each CSR, the left branch extracts deep feature maps with two cascaded SR modules. The right branch compresses the feature maps of base layer by virtue of downsampling. By this way, the extraction capacity of cross stages and the computation efficiency are both improved.

In the Neck, the shallow angular features are enhanced by the deep semantic features of sources through reverse iterative fusion. Feature maps with higher resolution but semantically stronger are generated by the CBF and UPS modules, which are then merged with the feature maps from corresponding CSR module on the same scale. By virtue of this design, features are extracted and fused iteratively from deep to shallow via upsampling and concatenation operations.

In the Head, DoAs of sources with confidence scores are predicted directly. In particular, a $3 \times 1$ Conv and a $1 \times 1$ Conv are used to transform the output feature maps of Neck into a tensor $\hat{\chi} \in \mathbb{R}^{24 \times P \times 5}$. Each attribute of $\hat{\chi}$ is the same as that of $\chi$. In order to accelerate the convergence speed of network, the location coordinates of the slice $\hat{\chi}^{i, j, 1: 4}$ are offsets relative to the location of SubReg. A postprocess procedure is required to obtain the true angular coordinates.

\section{DoAs prediction of sources}

We use sigmoid function $\zeta(\cdot)$ to constrain the network's predictions $\hat{\chi}$ to fall in the range of 0 and 1 . Particularly, there are 5 entries predicted from $j$-th MicroReg in the $i$-th SubReg, which are denoted as $x_{i, j}, y_{i, j}, w_{i, j}, h_{i, j}, \kappa_{i, j}$, respectively. Then, the predicted box is defined as $b \hat{o} x_{i, j}$ $\triangleq\left\{\hat{b}_{x_{i, j}}, \hat{b}_{y_{i, j}}, \hat{b}_{w_{i, j}}, \hat{b}_{h_{i, j}}, \hat{b}_{\kappa_{i, j}}\right\}$, where

$$
\begin{aligned}
\hat{b}_{x_{i, j}} & =\left(\zeta\left(x_{i, j}\right)+(i-1)\right) \times \Delta \\
\hat{b}_{y_{i, j}} & =\zeta\left(y_{i, j}\right) \\
\hat{b}_{w_{i, j}} & =W \cdot e^{w_{i, j}} \\
\hat{b}_{h_{i, j}} & =e^{h_{i, j}} \\
\hat{b}_{\kappa_{i, j}} & =\zeta\left(\kappa_{i, j}\right)
\end{aligned}
$$

In (4), $i=1,2, \cdots, S$ and $j=1,2, \cdots, P . \Delta$ is the angle coverage for each SubReg. If $S=24$ and $P=3$, then $\Delta=8$ and there are 72 predicted boxes in total. As many predicted boxes map to the same incident direction, the soft non maximum suppression (soft-NMS) algorithm [19] is applied to eliminate the redundant boxes so as to obtain DoAs of sources. Moreover, the slice $\hat{\chi}^{i, j,:}$ of network's predictions $\hat{\chi}$ is assigned with $b \hat{o} x_{i, j}$. Then the updated $\hat{\chi}$ is applied to evaluate the loss function during the training.

\section{Loss function}

The training of YOLO-DoA is performed offline in a supervised manner over the training dataset $\mathbb{D}_{\text {train }}$. We attempt

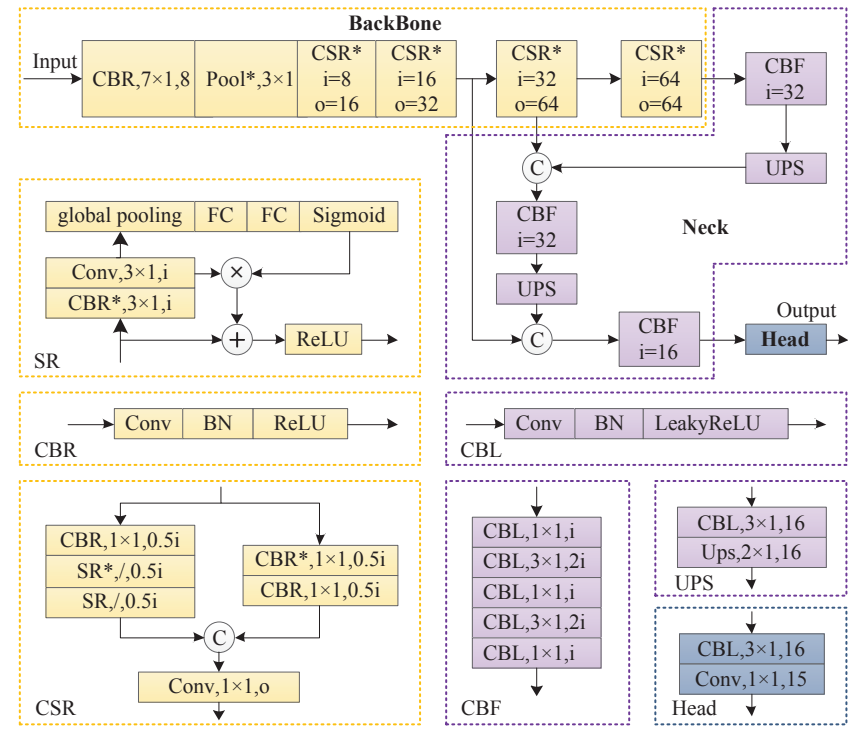

Fig. 2: The block diagram of the YOLO-DoA.

to optimize the set of all trainable parameters $\boldsymbol{\vartheta}$ whose updates are carried out via back-propagation by minimizing the reconstruction error, viz.,

$$
\boldsymbol{\vartheta}^{\text {opt }}=\underset{\boldsymbol{\vartheta}}{\arg \min } \frac{1}{\left|\mathbb{D}_{\text {train }}\right|} \sum_{(\overline{\mathbf{x}}, \boldsymbol{\chi}) \in \mathbb{D}_{\text {train }}} \operatorname{Loss}(\chi, \hat{\boldsymbol{\chi}})
$$

In (5), the loss function is composed of confidence loss and regression loss. Particularly, the weighted cross-entropy function is adopted as the confidence loss. The regression loss is evaluated by the GIoU function [15] between box $_{i, j}$ and the predicted boxes $\hat{b o} x_{i, j}$. Loss $(\chi, \hat{\chi})$ is expressed as

$$
\begin{aligned}
& \operatorname{Loss}(\chi, \hat{\chi})=-\sum_{i=1}^{S} \sum_{j=1}^{P}\left(1-\hat{\chi}^{i, j, 5}\right)^{\gamma} \chi^{i, j, 5} \log \zeta\left(\hat{\chi}^{i, j, 5}\right) \\
& -\sum_{i=1}^{S} \sum_{j=1}^{P} \mathbb{I}_{i, j}^{\mathrm{nobj}}\left(1-\hat{\chi}^{i, j, 5}\right)^{\gamma} \log \left(1-\zeta\left(\hat{\chi}^{i, j, 5}\right)\right) \\
& +\lambda_{\text {coord }} \sum_{i=1}^{S} \sum_{j=1}^{P} \chi^{i, j, 5}\left(1-\operatorname{GIoU}\left(\chi^{i, j, 1: 4}, \hat{\chi}^{i, j, 1: 4}\right)\right)
\end{aligned}
$$

where $S$ and $P$ are the total number of SubRegs and MicroRegs, respectively. $\gamma$ is the weighted factor. If GIoU ratios between the predicted box $b \hat{o} x_{i, j}$ and all of the angular boxes are less than threshold value $\tau$, the $\mathbb{I}_{i, j}^{\text {nobj }}$ is 1 , Otherwise $\mathbb{I}_{i, j}^{\text {nobj }}=0 . \lambda_{\text {coord }}$ is the penalty factor of regression loss.

\section{EXPERIMENTAL RESULTS AND ANALYSIS}

We consider an ULA-5 at half-wavelength distance. Two sources with a center frequency of $433 \mathrm{MHz}$ are impinged on the array from far field. To possibly simulate a real-word wireless communication, the maximum amplitude error and maximum phase error between channels are within $1 \mathrm{~dB}$ and $10^{\circ}$, respectively. The Gaussian noise ranges from $0 \mathrm{~dB}$ to $18 \mathrm{~dB}$ with step size of $3 \mathrm{~dB}$. For each SNR value, 642,000 samples generated on integer grids in the range of $\left[-90^{\circ}\right.$ 
$90^{\circ}$ ) are formed as training dataset $\mathbb{D}_{\text {train }}$. 192,600 samples

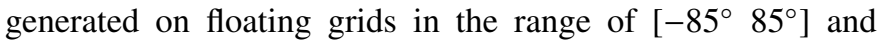
$\left[-90^{\circ} 90^{\circ}\right)$ are formed as test datasets $\mathbb{D}_{\text {test } \pm 85}$ and $\mathbb{D}_{\text {test } \pm 90}$, respectively. For each sample, 128 snapshots of array are utilized. The empirical RMSE metric in [9] is employed to evaluate the accuracy of YOLO-DoA and the counterparts.

During the training, the Adam optimizer is used, in which the initial learning rate is 0.01 (reduce by $4 \%$ after each epoch). The mini-batch size is 200 . In loss calculation, $\lambda_{\text {coord }}=$ 2, $\gamma=2$, and $\tau=0.5$. All experiments are conducted on the tensorflow-gpu 1.13.1 version, CUDA 10.0, two Intel Xeon E5-2678v3@2.50GHz CPUs and 128GB RAM and NVIDIA GeForce RTX2080.

\section{A. Parameters selection for data labeling}

The width of angular box $W$ can affect the accuracy of DoA estimation. When $W$ is too large, more irrelevant features are imposed during the training, which hinders the learning of angular features. On the contrary, multiple predicted boxes with approximated confidence scores are generated for the same incident direction, which reduce the effectiveness of softNMS. Hence, we evaluate the RMSE of YOLO-DoA with respect to $W$ given $\mathrm{SNR}=9 \mathrm{~dB}$ and $P=3$. As shown in Fig. $3(\mathrm{a})$, we can see that the optimal value of $W$ is $2^{\circ}$.
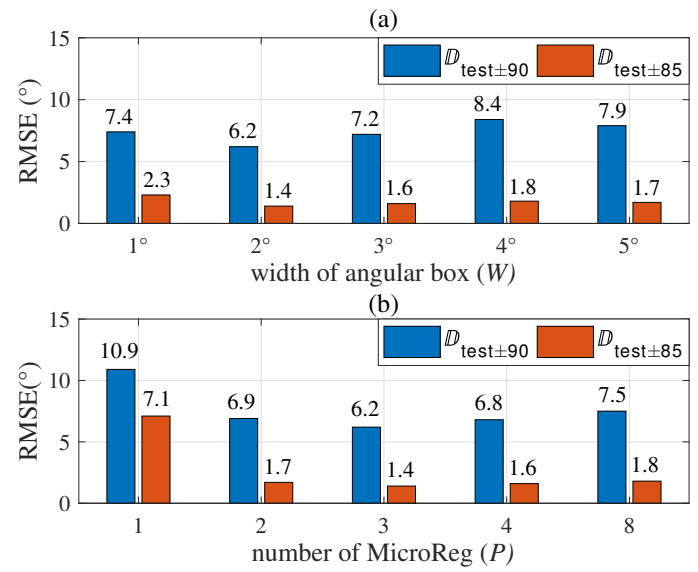

Fig. 3: The RMSEs against $W$ and $P$

Except for the width of angular box, the number of MicroRegs $P$ can also affect the accuracy of DoA estimation, we further evaluate the RMSE versus $P$ given $\mathrm{SNR}=9 \mathrm{~dB}$ and $W=2^{\circ}$, the results of which are shown in Fig. 3(b). We can see that $P=3$ is optimal. That's because when too few MicroRegs are utilized, the sources with small spatial spacing cannot be separated rightly. Instead, too many MicroRegs will produce more redundant predicted boxes, which also reduces the effectiveness of soft-NMS.

\section{B. The effectiveness verification of YOLO-DoA}

Given $\mathrm{SNR}=9 \mathrm{~dB}, W=2^{\circ}$ and $P=3$, we illustrate the effectiveness of YOLO-DoA in an incremental manner in this experiment. Simulation results are shown in TABLE I, where GFLOPs [12] and MPS (mini-batches per second) denote the computational cost and the prediction speed, respectively. The Para. denotes the trainable parameters of network [10]. The former and later items in RMSE are evaluated on test datasets $\mathbb{D}_{\text {test } \pm 85}$ and $\mathbb{D}_{\text {test } \pm 90}$, respectively.

TABLE I: The effectiveness study of YOLO-DoA.

\begin{tabular}{|c|c|c|c|c|c|}
\hline & Methods & Para. & GFLOPs & MPS & RMSE \\
\hline A & YOLO-Basic & $22.391 \mathrm{M}$ & 81.041 & 1.74 & $1.9^{\circ}, 6.3^{\circ}$ \\
\hline B & YOLO-ResNet18 & $5.496 \mathrm{M}$ & 18.649 & 3.61 & $1.9^{\circ}, 7.2^{\circ}$ \\
\hline C & YOLO-ResNet18 & $0.162 \mathrm{M}$ & 0.721 & 8.22 & $2.3^{\circ}, 7.6^{\circ}$ \\
\hline D & + CSP Connection & $0.080 \mathrm{M}$ & 0.332 & 8.53 & $2.2^{\circ}, 7.5^{\circ}$ \\
\hline E & + GIoU Loss & $0.080 \mathrm{M}$ & 0.332 & 8.23 & $1.5^{\circ}, 6.5^{\circ}$ \\
\hline F & + SE Operation & $0.081 \mathrm{M}$ & 0.333 & 8.08 & $1.4^{\circ}, 6.2^{\circ}$ \\
\hline
\end{tabular}

A: Based on YOLOv3 [12], the Heads with medium scale and large scale are removed except for the small scale Head. In the convolution layers, the kernel size $3 \times 3$ and the stride of $2 \times 2$ are adjusted to $3 \times 1$ and $2 \times 1$, respectively. The rest parts of the network structure are unchanged, which form the YOLO-Basic. The performance of YOLO-Basic illustrates that it is feasible to take DoA estimation as a detection problem. However, it shows that YOLO-Basic has two drawbacks including large network size and high computational cost.

$\mathrm{A} \rightarrow \mathrm{B}$ : In the YOLO-ResNet18, we replace the backbone of YOLO-Basic with ResNet18 [14]. The kernel size and stride of each Conv in ResNet18 are handled in the same way as YOLO-Basic. Obviously, the RMSE is almost unchanged while the network size and computational cost are reduced.

$\mathrm{B} \rightarrow \mathrm{C}$ : In the last layer of ResNet18, we shrink the number of channels from 512 to 256 . Moreover, the number of channels in each Conv is compressed with a factor of 4 . Both network size and computational cost of the YOLO-ResNet18 ${ }^{\dagger}$ are significantly reduced and the prediction speed is also improved. But the RMSE is increased to a certain extent.

$\mathrm{C} \rightarrow \mathrm{F}$ : Next, the CSP connections (D) are imposed into the YOLO-ResNet $18^{\dagger}$ to improve the whole performance. And then, we replace the regression loss with GIoU loss function (E) to enhance the utilization about features. In the step F, channel-wise attention mechanism is utilized by using the SE operation, which improves the robustness of network.

Through steps $\mathrm{A} \rightarrow \mathrm{F}$, the construction of YOLO-DoA is completed. Compared to YOLO-Basic, both the parameters and computational cost of YOLO-DoA are reduced by $99.6 \%$. Meanwhile, the prediction speed is increased by a factor of 4.6 and RMSE is decreased obviously. Therefore, the effectiveness of YOLO-DoA is confirmed.

\section{Comparison to other data-driven methods}

In this experiment, we summarize the methods that we used in the comparison to YOLO-DoA, including ResNet-DoA [8], CNN-DoA [9], and RF-DoA [10]. Additionally, we calculate the Cramer-Rao low bound (CRLB) for the correlated sources [20]. The RMSEs of different methods against SNR values are shown in Fig. 4(a) and Fig. 4(b), which are evaluated within range of $\left[-85^{\circ} 85^{\circ}\right]$ and $\left[-90^{\circ} 90^{\circ}\right)$, respectively.

As reported in Fig. 4(a), we can see that YOLO-DoA shows superiority in term of RMSE compared to the counterparts. That's because the binary vector labels adopted in classification network exploits line-type local features only. However, 

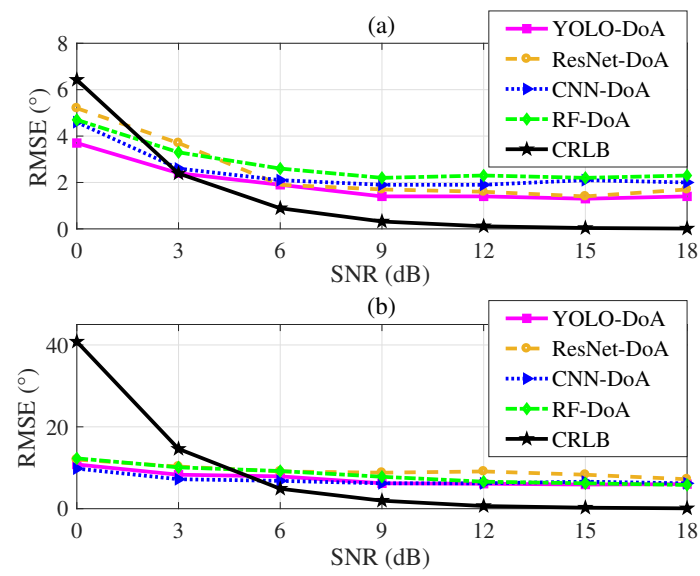

Fig. 4: The RMSE of different methods against the SNR.

the area-type features are utilized in YOLO-DoA. Moreover, as the peaks-search procedures adopted in the counterparts are no longer needed in YOLO-DoA, the fit-error is eliminated. In addition, it shows that DoA estimation with data-driven methods are biased at SNR $=0 \mathrm{~dB}$, because the RMSEs of which are smaller than CRLB. Besides, according to the results of Fig. 4(b), when the DoAs of sources are close to $\pm 90^{\circ}$, RMSEs of all data-driven methods are deteriorated.

To illustrate the advantages of YOLO-DoA, we also compare it to the counterparts about key assessing factors in terms of network size, the computational cost, prediction speed as well as the corresponding inference time, respectively.

TABLE II: Performance comparison of different methods.

\begin{tabular}{|c|c|c|c|c|}
\hline Methods & Para. & GFLOPs & BPS & InferTime \\
\hline YOLO-DoA & $\mathbf{0 . 0 8 1 M}$ & $\mathbf{0 . 3 3 3}$ & $\mathbf{8 . 0 8}$ & $\mathbf{1 2 3 . 7 m s}$ \\
\hline ResNet-DoA [8] & $3.039 \mathrm{M}$ & 8.068 & 0.98 & $1022.5 \mathrm{~ms}$ \\
\hline CNN-DoA [9] & $15.668 \mathrm{M}$ & 5.743 & 0.51 & $1978.7 \mathrm{~ms}$ \\
\hline RF-DoA [10] & $0.083 \mathrm{M}$ & 4.609 & 0.40 & $2498.9 \mathrm{~ms}$ \\
\hline
\end{tabular}

As shown in TABLE II, the parameters of YOLO-DoA are approximately equivalent to those of RF-DoA and far less than those of ResNet-DoA and CNN-DoA, which demonstrates that it is a lightweight network. Additionally, YOLO-DoA has the lowest computational cost and the fastest prediction speed compared to the counterparts, and it's the best one to meet the requirements for real-time processing of multi-batch data per second. Therefore, YOLO-DoA is a cost-efficient method while obtaining a state-of-the-art DoA estimation accuracy.

\section{CONCLUSIONS}

In this correspondence, a novel end-to-end neural network for direction finding on the basis of YOLOv3 is proposed. It has several merits including small network size, low computational cost, real-time prediction speed, and high accuracy of DoA estimation. Different from existing methods based on classification neural networks, YOLO-DoA performs the DoA estimation by regarding it as a detection problem to spatially separated angular boxes. Experimental results illustrate that it achieves a better performance than the counterparts, which can be used in small unmanned aerial vehicles (small-UAVs) localization, wireless communications, radio frequency jamming countermeasures, etc. The source code is available at https://github.com/fanrongca/YOLO-DoA.

\section{REFERENCES}

[1] W. Miao, C. Luo, G. Min and Z. Zhao, "Lightweight 3-D beamforming design in 5G UAV broadcasting communications," IEEE Trans. Broadcast., vol. 66, no. 2, pp. 515-524, Jun. 2020.

[2] F. Wen, P. Liu, H. Wei, Y. Zhang and R. C. Qiu, "Joint Azimuth, Elevation, and Delay Estimation for 3-D Indoor Localization," IEEE Trans. Veh. Technol., vol. 67, no. 5, pp. 4248-4261, May 2018.

[3] S. Uehashi, Y. Ogawa, T. Nishimura and T. Ohgane, "Prediction of TimeVarying Multi-User MIMO Channels Based on DOA Estimation Using Compressed Sensing, IEEE Trans. Veh. Technol., vol. 68, no. 1, pp. 565577, Jan. 2019.

[4] Z. Guo, X. Wang and W. Heng, "Millimeter-wave channel estimation based on 2-D beamspace MUSIC method," IEEE Trans. Wirel. Commun., vol. 16, no. 8, pp. 5384-5394, Aug. 2017.

[5] D. Ng, E. S. Lo and R. Schober, "Robust beamforming for secure communication in systems with wireless information and power transfer," IEEE Trans. Wirel. Commun., vol. 13, no. 8, pp. 4599-4615, Aug. 2014.

[6] R. Roy and T. Kailath, "ESPRIT-estimation of signal parameters via rotational invariance techniques," IEEE Trans. Acoust. Speech Signal Process., vol. 37, no. 7, pp. 984-995, Jul. 1989.

[7] L. X. Wang and J. M. Mendel, "Fuzzy basis functions, universal approximation, and orthogonal least-squares learning," IEEE Trans. Neural Netw., vol. 3, no. 5, pp. 807-814, Sept. 1992.

[8] R. Fan, C. Si, H. Guo, Y. Wan, Y. Xu, "Direction finding for coherent sources with deep hybrid neural networks," Int. J. Electron., 2021.

[9] G. K. Papageorgiou, M. Sellathurai and Y. C. Eldar, "Deep networks for direction-of-arrival estimation in low SNR,' IEEE Trans. Signal Process., vol. 69, pp. 3714-3729, Jun. 2021.

[10] R. Akter, V. Doan, T. Huynh-The and D. Kim, "RFDOA-Net: An efficient convNet for RF-based DOA estimation in UAV surveillance systems," IEEE Trans. Veh. Technol., vol. 70, no. 11, pp. 12209-12214, Nov. 2021.

[11] G. Tang and A. Nehorai, "Performance analysis for sparse support recovery," Trans. Inf. Theory., vol. 56, no. 3, pp. 1383-1399, Mar. 2010.

[12] J. Redmon and A. Farhadi, "Yolov3: An incremental improvement," arXiv e-prints, 2018. [Online]. Available: https://arxiv.org/abs/1804.02767

[13] C. Wang, H. Mark Liao, Y. Wu, P. Chen, J. Hsieh and I. Yeh, "CSPNet: A new backbone that can enhance learning capability of CNN," IEEE/CVF Conf. CVPRW., Jun. 2020, pp. 1571-1580.

[14] K. He, X. Zhang, S. Ren and J. Sun, "Deep residual learning for image recognition," IEEE Conf. CVPR., Jun. 2016, pp. 770-778.

[15] H. Rezatofighi, N. Tsoi, J. Gwak, A. Sadeghian, I. Reid and S. Savarese, "Generalized intersection over union: a metric and a loss for bounding box regression," IEEE/CVF Conf. CVPR., Jun. 2019, pp. 658-666.

[16] R. Fan, X. Zhu, W. Tang and C. Si, "MFFNet: Multi-path Features Fusion Network for Source Enumeration," IEEE Communications Lett., doi: 10.1109/LCOMM.2021.3139676.

[17] Y. Yuan, S. Wu, M. Wu and N. Yuan, "Unsupervised learning strategy for direction-of-arrival estimation network," IEEE Signal Process. Lett., vol. 28, pp. 1450-1454, Jul. 2021.

[18] A. Krizhevsky, I. Sutskever, and G. E. Hinton, "Imagenet classification with deep convolutional neural networks," Commun. ACM., vol. 60, no. 6, pp. 84-90, Jun. 2017.

[19] N. Bodla, B. Singh, R. Chellappa and L. S. Davis, "Soft-NMS Improving object detection with one line of code," IEEE Conf. ICCV., Oct. 2017, pp. 5562-5570.

[20] P. Stoica and A. Nehorai, "Performance study of conditional and unconditional direction-of-arrival estimation," IEEE Trans. Acoust. Speech Signal Process., vol. 38, no. 10, pp. 1783-1795, Oct. 1990. 